\title{
ExSENINESEGES \\ Estimativa dos componentes genéticos do peso ao nascimento, em um rebanho ovino com diferentes graus de sangue Polwarth
}

\author{
[Estimation of genetic components of birth weight, in an ovine herd with different levels of \\ Polwarth blood]
}

\section{"Artigo Científico/Scientific Article"}

\author{
Fernando Amarilho Silveira ${ }^{1 *}$, Nelson José Laurino Dionello ${ }^{1}$, Gilson de Mendoça ${ }^{2}$, \\ Marvyn da Silveira Ribeiro ${ }^{3}$, Nicholas da Silveira Silva ${ }^{1}$, Amanda Weber Garcia ${ }^{1}$
}

\author{
${ }^{1}$ Departamento de Zootecnia, Universidade Federal de Pelotas, Pelotas-RS, Brasil. \\ ${ }^{2}$ Departamento de Fisiologia, Universidade Federal de Pelotas, Pelotas-RS, Brasil. \\ ${ }^{3}$ Agropecuário São João, Herval-RS, Brasil. \\ *Autor para correspondência/Corresponding author: E-mail: amarillo@zootecnista.com.br
}

\begin{abstract}
Resumo
O peso ao nascimento é uma característica relacionada diretamente com a sobrevivência pós-natal, em que cordeiros com pesos extremos (leves ou pesados) são mais susceptíveis à morte. Logo, o conhecimento dos fatores genéticos e ambientais que influenciam tal caraterística, em rebanhos mestiços, ainda são obscuros. Neste sentido, objetivou-se verificar os parâmetros genéticos para a característica de peso ao nascer, pelo método da máxima verossimilhança restrita, com base em 1033 registros de nascimento de um rebanho mestiço com diferentes graus de sangue Polwarth. Assim, testou-se 12 modelos animal, sendo o modelo que considerou os efeitos aditivos direto, materno e a covariância entre ambos, o que melhor explicou a variação fenotípica. Para este, foram obtidos coeficientes de herdabilidade direta, materna e a correlação entre tais efeitos de 0,20, 0,54 e $-0,69$, respectivamente. Assim, verifica-se baixos coeficientes de herdabilidade direta e altos para o efeito materno, como uma alta correlação negativa entre os efeitos direto e materno.
\end{abstract}

Palavras-chave: avaliação genética; cruzamento; máxima verossimilhança restrita; melhoramento genético.

\begin{abstract}
Birth weight is a characteristic directly related to postnatal survival, in which lambs with extreme weights (light or heavy) are more susceptible to death. Therefore, the knowledge of the genetic and environmental factors influencing this trait in mixed-breed herds is still obscure. In this sense, we aimed to verify the genetic parameters for the birth weight trait, by the method of the maximum restricted likelihood, based on 1033 records of the birth of a mixed-breed herd with different degrees of Polwarth blood. Thus, we tested 12 animal models, being the model that considered direct, maternal additive effects and covariance between both the one which best explained the phenotypic variation. For these, we obtained direct and maternal heritability coefficients and the correlation between these effects of $0.20,0.54$ and -0.69 , respectively. Thus, low direct and high heritability coefficients for the maternal effects is observed and a high negative correlation between direct and maternal effects.
\end{abstract}

Keywords: genetic evaluation; crossing; maximum likelihood restricted; genetic improvement.

\section{Introdução}

As obtenções de estimativas de valores genéticos para as características produtivas são de grande importância para nortear o sistema produtivo, assim possibilitando planejar os acasalamentos e selecionar animais mais

responsivos as práticas de manejo extensivo, para que se possa aumentar a eficiência dos sistemas de produção ovina (Ciappesoni et al., 2014; AmarilhoSilveira et al., 2017). No entanto, a falta de informações dos parâmetros genéticos, necessários 
para prever ganhos genéticos é comumente citada como um dos obstáculos. Desta forma, são necessárias tais estimativas, para determinar o método de seleção a ser utilizado, estimando o ganho genético máximo, que pode ser alcançado (Lôbo et al., 2009).

No Brasil, de modo geral, o baixo nível de escrituração zootécnica é uma realidade, consequentemente limitando a precisão das estimativas dos parâmetros genéticos em ovinos (Lôbo et al., 2014; Amarilho-Silveira et al., 2018). Assim, quando escriturada, a principal característica é o peso ao nascer, especialmente por estar relacionada com a sobrevivência dos neonatos, que segundo Banchero et al. (2012), cordeiros que nascem mais pesados apresentam maiores chances de sobrevivência quando comparados a cordeiros leves. Sawalha et al. (2007) descreveram que existe uma relação não linear bem estabelecida entre a sobrevivência do cordeiro e o peso ao nascer, ou seja, tanto cordeiros muito leves, como muito pesados estão em risco de morrer antes do desmame; enquanto que cordeiros com um peso intermediário terão uma melhor chance de sobrevivência. Cordeiros pequenos são propensos a sucumbir à hipotermia e inanição, enquanto cordeiros pesados estão em maior risco de morrer por causa de distocia.

Conforme o descrito por Lavvaf e Noshary (2008), essa característica, além de ser relacionada à sobrevivência, é a primeira acerca do crescimento do indivíduo, sendo sua expressão fenotípica na progênie, influenciada pela capacidade de nutrição intrauterina. Assim, a mãe contribui no peso ao nascer da progênie de duas maneiras: 1) pelos seus efeitos genéticos diretos passados para a progênie e 2) pela sua habilidade para proporcionar um ambiente uterino adequado.

No contexto produtivo nacional, a raça Polwarth, com orientação zootécnica de $70 \%$ para produção lã e $30 \%$ para produção de carne (ARCO, 2016), apresenta 345 rebanhos registrados junto a Associação Brasileira de Criadores de Ovinos, distribuídos em 61 municípios exclusivamente no Rio Grande do Sul (McManus et al., 2016). Logo, produtores estão lançando mão de tecnologias para o aumento produtivo desta raça. Para Ganzábal et al. (2012) a inclusão de raças como a Finnish Landrace, a Frisona Milchschaf ou o produto destas (F1) nos rebanhos tradicionalmente criados em determinadas regiões, pode ser uma alternativa para incrementar precocidade sexual, taxa ovulatória, velocidade de parto, produção de leite, velocidade de crescimento e outros atributos.

Contudo, o desconhecimento do impacto genético na inclusão de reprodutores $\mathrm{F} 1$ das raças Finnish Landrace e Frisona Milchschaf nas raças tradicionalmente criadas no Rio Grande do Sul, cria um anseio à avaliação genética dos animais.

Conforme Safari et al. (2005), o desenvolvimento de avaliações genéticas eficazes e programas de melhoramento genético requerem o conhecimento dos parâmetros genéticos de cada característica, quais sejam economicamente importantes para a produção ovina ou de qualquer outra espécie com o mesmo fim. Assim, devido a disponibilidade de dados de peso ao nascimento de ovinos de um rebanho com diferentes graus de sangue Polwarth, o objetivo deste trabalho foi verificar os parâmetros genéticos e as tendências genéticas dos animais deste rebanho, verificando o modelo animal que melhor prediz os valores genéticos.

\section{Materiais e Métodos}

Foram disponibilizados 1033 registros de nascimento de um rebanho com base materna a raça Polwarth e paterna o F1 das raças Finnish Landrace X Frisona Milchschaf, de 2012 até 2016, oriundos da Agropecuária São João situada no município de Herval, Rio Grande do Sul, Brasil, localizada nas coordenadas geográficas $32^{\circ} 03^{\prime} 21.2^{\prime \prime} \mathrm{S} ; 53^{\circ} 18^{\prime} 15.7^{\prime \prime W}$. Estes 1033 registros de nascimentos foram utilizados para os cálculos de estimativas dos parâmetros genéticos.

$\mathrm{O}$ arquivo de pedigree foi composto por nove pais e 345 mães, totalizando 1409 animais (progênies, pais e avós). No ano de 2012, quando se iniciou a escrituração zootécnica do peso ao nascimento, os cordeiros nascidos não tinham registro genealógico paterno, logo decidiu-se utilizar esses dados como registro produtivo materno. A partir do ano 2013 começou os registros da característica referente ao cruzamento Polwarth X F1 (Finnish Landrace X Frisona Milchschaf), sendo os produtos tratados como F2.

No ano de 2014 algumas fêmeas F2, nascidas em 2013, produziram seus produtos (F3), sendo estes filhos de um carneiro da raça Texel. Em 2015 foram reproduzidas as F2 nascidas em 2013 e algumas nascidas em 2014, também com um carneiro da raça Texel.

Devido a esses cruzamentos e alguns retrocruzamentos (F2 e F3), foi considerado a composição racial dos indivíduos nascidos na 
propriedade como efeito aleatório, conforme o modelo testado. Sendo filhos(as) das ovelhas Polwarth sem registro paterno e com indefinição racial considerados Meio Sangue Base; filhos(as) das ovelhas Polwarth sem registro paterno com padrão racial, foram considerados Base; filhos(as) das ovelhas Polwarth com carneiros Texel considerados Base X Texel; filhos(as) das ovelhas Polwarth com carneiros F1 considerados F2; filhos(as) das ovelhas F2 sem registro paterno, foram considerados Meio Sangue F2; filhos(as) das F2 com carneiros F1 considerados F2 X F1; e filhos(as) das F2 com carneiros Texel considerados F2 X Texel.

Os grupos de contemporâneos (GC) foram formados conforme o mês e o ano de nascimento, o sexo e o tipo de parto. Como efeitos fixos (efeitos sistemáticos) foi considerado o GC, a idade da mãe ao parto (oito níveis - de um a sete anos e idade não conhecida) e a interação entre eles. Foram eliminados registros que apresentavam valores acima e abaixo de três desvios-padrão da média populacional e que estavam em GC com menos de cinco animais. Assim, de 1033 animais registrados inicialmente, apenas 950 registros foram utilizados, distribuídos em 39 GC.

Testou-se a influência dos efeitos sistemáticos no peso ao nascimento, utilizando o método dos mínimos quadrados pelo pacote estatístico R (R Core Team, 2018). Logo verificouse apenas efeito significativo $(\mathrm{P}<0,05)$ para o $\mathrm{GC}$ (Tabela 1), consequentemente, a idade da mãe ao parto e a interação entre o GC e a idade da mãe ao parto não foram considerados nos modelos.

Tabela 1. Análise descritiva dos dados e dos efeitos fixos para a característica de peso ao nascimento, em ovinos mestiços criados no Rio Grande do Sul.

\begin{tabular}{|c|c|c|c|c|}
\hline \multicolumn{5}{|c|}{ Análise descritiva dos dados } \\
\hline \multirow{4}{*}{$\begin{array}{c}\text { Observações (n) } \\
950\end{array}$} & Média $(\mathrm{Kg}) \quad \mathrm{I}$ & Desvio padrão (Kg) & Mínimo $(\mathrm{Kg})$ & Máximo $(\mathrm{Kg})$ \\
\hline & 3,960 & 0,860 & 1,400 & 6,540 \\
\hline & \multicolumn{3}{|c|}{ Análises dos efeitos fixos } & \\
\hline & Número de níveis & s $\quad$ Probabilida & & \\
\hline$G C$ & 39 & $\mathrm{P}<0,001$ & & \\
\hline Idade da mãe ao parto (Id) & 8 & $\mathrm{P}=0,471$ & & \\
\hline$G C X I d$ & 312 & $P=0,908$ & & \\
\hline
\end{tabular}

${ }^{1} \mathrm{PN}$ - Peso ao nascimento.

${ }^{2}$ Efeito significativo $(\mathrm{P} \leq 0,05)$ pelo teste de mínimos quadrados.

Após a organização dos dados e a determinação do efeito sistemático, foram testados 12 modelos com fins de determinar o melhor modelo de predição dos valores genéticos, ou seja, escolha do modelo que melhor ajustou os dados. Os 12 modelos testados foram:

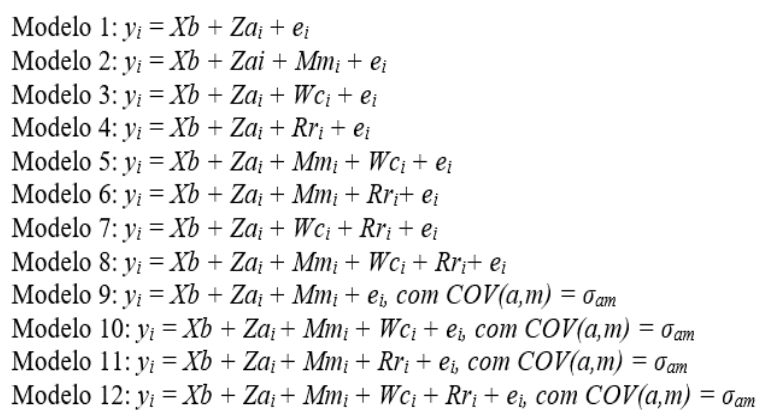

Em que $y$ é uma observação no animal $i$ como a soma dos efeitos ambientais $b$; variância genética aditiva direta $a$; variância genética aditiva materna $m$; ambiente permanente materno $c$; composição racial $r$; fatores desconhecidos, residuais ou erro $e$; e a covariância entre efeitos diretos e maternos. As letras $X, Z, M, W$ e $R$ sãos as matrizes de incidência para os efeitos fixos, diretos, maternos e de ambiente permanente materno, respectivamente. $\sigma_{a m}$ é a matriz de covariância entre os efeitos diretos e maternos.

As análises foram realizadas utilizando o método da máxima verossimilhança restrita (REML) sob um modelo animal, considerando a característica de peso ao nascimento, empregando o programa WOMBAT (Meyer, 2016). Para a escolha do melhor modelo utilizou-se o critério de AIC (Akaike, 1977), o qual foi calculado para classificar os modelos de acordo com sua capacidade de ajustar os dados, em que quanto menor é o valor de AIC, melhor é o ajuste. O cálculo do AIC foi dado pela fórmula:

$A I C_{i}=-2 \log L_{i}+2 p_{i}$

Sendo o $\log L_{i}$ é o logaritmo da máxima verossimilhança do modelo $i$ em convergência e $p_{i}$ é o número de parâmetros estimados para cada modelo.

Após a determinação do modelo que melhor ajustou os dados, foram estimados os componentes da (co)variância e os parâmetros genéticos. As 
tendências genéticas foram calculadas pelos valores genéticos médios de cada ano (2012 a 2016), e submetidos a análise de regressão linear pelo pacote estatístico R (R Core Team, 2018). Os ganhos genéticos e fenotípicos foram obtidos pela diferença dos valores genéticos e fenotípicos entre 2012 e 2016.

\section{Resultados e Discussão}

O modelo 9 foi o que melhor ajustou os dados pelo critério AIC, para a característica de peso ao nascimento, qual considerou os efeitos direto e materno e a covariância entre ambos (Tabela 2). Dados que concordaram com os obtidos por Kariuki et al. (2010), Di et al. (2011), Shokrollahi e Baneh (2012), Ulutas et al. (2013) e Boujenane e Diallo (2017), que pesquisaram ovinos da raça Dorper, Merino Chinês superfino, Arabi, Karayaka e Sardi, respectivamente.

Nos estudos de Bahreini Behzadi et al. (2007), Eskandarinasab et al. (2010) e Jafaroghli et al. (2010), respectivamente, com as raças Kermani, Afshari e Moghani, o modelo que melhor estimou os parâmetros genéticos, para a característica em questão, foi o que considerou os efeitos direto e materno. Já Gowane et al. (2010a) para ovinos da raça Merino Bharat, Gowane et al. (2010b) e Prakash et al. (2012) para ovinos Malpura, Mohammadi et al. (2013) para ovinos Makooei, Mirhoseini et al. (2015) para ovinos Karakul e, Amarilho-Silveira et al. (2018) para ovinos Texel, encontraram melhor ajuste com o modelo que considerou os efeitos aleatórios diretos e de ambiente permanente materno.

Com ovinos da raça Mehraban, Lori, Morada Nova, D'man e Santa Inês, respectivamente, Mohammadi e Endris (2007), Lavvaf e Noshary (2008), Shiotsuki et al. (2014), Boujenane et al. (2015) e Aguirre et al. (2016) estimaram os parâmetros genéticos utilizando o modelo que considerou os efeitos diretos, maternos e de ambiente permanente materno. Para ovinos das respectivas raças, Dorper, Columbia, Targhee, Mehraban, Baluchi, Muzaffarnagari, Baluchi e Lori, foram estimados os parâmetros genéticos utilizando o modelo que considerou os efeitos animais (direto e materno), a covariância entre eles e o ambiente permanente materno (Neser et al., 2001, Hanford et al., 2002, Hanford et al., 2003, Zamani e Mohammadi, 2008, Abbasi et al., 2012, Mandal et al., 2015, Gholizadeh e Ghafouri-Kesbi, 2015 e Mohammadi et al., 2015).
Em ovinos de variada composição racial, na Nova Zelândia, o modelo que considerou os efeitos direto, materno e o ambiente temporário da mãe por ano (não testado no presente estudo) foi o que melhor ajustou os dados (Everett-Hincks et al., 2014).

Para a raça Lleyn, os componentes da (co)variância e os parâmetros genéticos, foram estimados por dois modelos, sendo considerado, no primeiro, os efeitos animais, a covariância entre eles e o efeito do ambiente permanente materno (Ceyhan et al., 2015). O segundo foi composto por todos os efeitos anteriores menos a covariância entre os efeitos direto e materno. Nas raças Dormer e Ile de France, o melhor modelo foi o que considerou efeitos direto e materno, a covariância entre ambos e o ambiente permanente materno (Zishiri et al., 2014). No entanto, no mesmo estudo, para a raça Merino Landsheep, apenas o efeito direto constou no modelo de melhor ajuste.

Na Tabela 2 são mostrados os componentes da (co)variância e os parâmetros genéticos estimados utilizando o Modelo 9, qual apresentou melhor ajuste pelo critério de AIC. Assim as variâncias aditiva direta, aditiva materna e a covariância entre os efeitos direto e materno foram, respectivamente, $0,122,0,322$ e $-0,130$. Os coeficientes de herdabilidades direta e materna foram 0,20 e 0,54 , respectivamente, e com uma correlação entre os efeitos direto e materno de 0,69 . A estimativa do coeficiente de herdabilidade direta apresentou valores próximo ao obtido por Kariuki et al. (2010), porém inferior aos obtidos por Shokrollahi e Baneh (2012) e Ulutas et al. (2013) e, superior aos obtidos por e Di et al. (2011) e Boujenane e Diallo (2017).

Nos estudos de Shokrollahi e Baneh (2012) e Ulutas et al. (2013), os coeficientes de herdabilidade direta foram, respectivamente, $0,42 \mathrm{e}$ 0,55 . Logo, a baixa herdabilidade direta $(0,07)$ encontrada por Boujenane e Diallo (2017), foi atribuída pelos mesmos a uma provável influência da baixa qualidade das pastagens em que esses animais eram mantidos, resultando em uma grande influência ambiental na expressão da característica. Já no presente trabalho não se identificou essa acentuada influência ambiental, em que os efeitos genéticos foram responsáveis por mais de $50 \%$ na variação no peso ao nascimento. No entanto, a variação genética, identificada neste trabalho, foi mais dependente do efeito materno do que propriamente do efeito direto. 
Assim, o coeficiente de herdabilidade materna, apresentado no modelo 9 da Tabela 2, foi superior aos obtidos por Kariuki et al. (2010), Shokrollahi e Baneh (2012), Di et al. (2011), Ulutas et al. (2013) e, Boujenane e Diallo (2017). No entanto, a correlação entre os efeitos animais obtida neste trabalho foi mais negativa do que os encontrados por Kariuki et al. (2010), Di et al. (2011), Ulutas et al. (2013) e, Boujenane e Diallo (2017), que obtiveram correlações na ordem de $0,47,-0,55,-0,46-0,60$, respectivamente. Logo, quando comparado com a correlação entre os efeitos direto e materno no estudo de Shokrollahi e Baneh (2012), o obtido na presente pesquisa foi menos negativa $(-0,69$ vs. $-0,87)$. Este antagonismo entre os efeitos diretos e maternos pode estar atrelado ao baixo número de registros, falta de informações no pedigree, a correlação entre os efeitos ambientais e a seleção natural (Di et al., 2011; Shokrollahi e Baneh, 2012). Segundo Boujenane e Diallo (2017), do ponto de vista evolutivo, a correlação negativa evita o aumento ou diminuição aguda do peso ao nascimento, assim evitando problemas de distocia e baixa viabilidade.

Tabela 2. Componentes da (co)variância, parâmetros genéticos e AIC para a característica de peso ao nascimento de ovinos mestiços criados no Rio Grande do Sul.

\begin{tabular}{|c|c|c|c|c|c|c|c|c|c|c|c|c|}
\hline & ${ }^{1} \sigma_{a}$ & $\sigma_{\mathbf{m}}$ & $\sigma_{c}$ & $\sigma_{\mathrm{r}}$ & $\boldsymbol{\sigma}_{\mathrm{am}}$ & $\sigma_{\mathrm{e}}$ & $\mathbf{h}^{2}$ & $\mathbf{m}^{2}$ & $c^{2}$ & $\mathbf{r}^{2}$ & Corr $_{a m}$ & AIC \\
\hline Modelo 1 & 0,428 & - & - & - & - & 0,210 & $0,67 \pm 0,092$ & - & - & - & - & 467,702 \\
\hline Modelo 2 & 0,069 & 0,193 & - & - & - & 0,318 & $0,12 \pm 0,084$ & $0,33 \pm 0,055$ & - & - & - & 445,592 \\
\hline Modelo 3 & 0,130 & - & 0,168 & - & - & 0,288 & $0,22 \pm 0,128$ & - & $0,28 \pm 0,064$ & - & - & 454,636 \\
\hline Modelo 4 & 0,423 & - & - & 0,003 & - & 0,213 & $0,66 \pm 0,093$ & - & - & $0,01 \pm 0,015$ & - & 469,392 \\
\hline Modelo 5 & 0,069 & 0,194 & 0,001 & - & - & 0,318 & $0,12 \pm 0,085$ & $0,33 \pm 0,122$ & $0,00 \pm 0,113$ & - & - & 447,612 \\
\hline Modelo 6 & 0,067 & 0,192 & - & 0,001 & - & 0,319 & $0,11 \pm 0,086$ & $0,33 \pm 0,055$ & - & $0,00 \pm 0,011$ & - & 447,576 \\
\hline Modelo 7 & 0,132 & - & 0,168 & 0,001 & - & 0,287 & $0,22 \pm 0,131$ & - & $0,28 \pm 0,064$ & $0,00 \pm 0,013$ & - & 456,696 \\
\hline Modelo 8 & 0,067 & 0,194 & 0,001 & 0,001 & - & 0,319 & $0,11 \pm 0,087$ & $0,33 \pm 0,123$ & $0,00 \pm 0,114$ & $0,00 \pm 0,011$ & - & 449,200 \\
\hline Modelo 9* & 0,122 & 0,322 & - & - & $-0,130$ & 0,283 & $0,20 \pm 0,135$ & $0,54 \pm 0,137$ & - & - & $-0,69 \pm 0,254$ & 445,396 \\
\hline Modelo 10 & 0,122 & 0,549 & 0,001 & - & $-0,139$ & 0,283 & $0,20 \pm 0,134$ & $0,55 \pm 0,22$ & $0,00 \pm 0,129$ & - & $-0,69 \pm 0,257$ & 447,440 \\
\hline Modelo 11 & 0,122 & 0,321 & - & 0,001 & $-0,139$ & 0,283 & $0,20 \pm 0,136$ & $0,54 \pm 0,138$ & - & $0,00 \pm 0,013$ & $-0,70 \pm 0,255$ & 447,412 \\
\hline Modelo 12 & 0,122 & 0,326 & 0,001 & 0,001 & $-0,140$ & 0,283 & $0,20 \pm 0,136$ & $0,54 \pm 0,221$ & $0,00 \pm 0,130$ & $0,00 \pm 0,013$ & $-0,70 \pm 0,325$ & 449,460 \\
\hline
\end{tabular}

${ }^{1} \sigma_{\mathrm{a}^{-}}$variância aditiva direta; $\sigma_{\mathrm{m}}-$ variância aditiva materna; $\sigma_{\mathrm{c}}-$ variância do ambiente permanente materno; $\sigma_{\mathrm{r}}-$ variância da composição racial; $\sigma_{a m}$ - covariância entre os efeitos direto e materno; $\sigma_{a}$ - variância residual; $h^{2}$ - coeficiente de herdabilidade direta; $\mathrm{m}^{2}$ - coeficiente de herdabilidade materna; $\mathrm{c}^{2}$ - proporção da variância fenotípica devido ao ambiente permanente materno; $\mathrm{r}^{2}-$ proporção da variância fenotípica devido à composição racial; Corr $_{a m}$ - correlação entre os efeitos direto e materno e; AIC - critério de AIC.

*Modelo que melhor ajustou os dados pelo critério de AIC.

Utilizando, também, o método da máxima verossimilhança, porém com o modelo que considerou os efeitos animais, a covariância entre eles e o ambiente permanente materno, Neser et al. (2001), Hanford et al. (2003), Zamani e Mohammadi (2008), Abbasi et al. (2012), Mandal et al. (2015), Gholizadeh e Ghafouri-Kesbi (2015) e, Mohammadi et al. (2015) encontraram correlação positiva de $0,35,0,09,0,35,0,44,0,05$, 0,49 e 0,68 entre os efeitos direto e materno, respectivamente. Logo, Zishiri et al. (2014) para a raça Dormer encontraram uma correlação de $-0,91$ entre os efeitos animais, e $-0,10$ para a raça Ile de France. Os mesmos atribuem, ao obtido para a raça Dormer, que a alta correlação negativa é derivada da falha na partição da variação genética. Qual pode ter sido influenciada pelos baixos laços genéticos entre os anos, em que pais e mães entram e saem do esquema de avaliação genética aleatoriamente, comprometendo a conectividade entre os anos avaliados.

Uma positiva correlação entre os efeitos direto e materno vai acelerar a resposta a seleção, enquanto que uma correlação negativa diminui essa resposta. Segundo Abbasi et al. (2012), o número de mães sem registros pode ser uma fonte de viés para a correlação entre tais efeitos. No estudo destes autores houve uma boa conexão entre os registros das mães e os registros de progênies, sendo que $77 \%$ das matrizes tiveram seus próprios registros de desempenho.

As tendências genéticas para os efeitos direto, materno e a variação fenotípica são mostrados na Figura 1. Como se observa, a tendência genética direta apresentou um incremento, com ganho genético de $0,027 \mathrm{~kg}\left(\mathrm{R}^{2}=\right.$ 0,42 ) entre os anos de 2012 a 2016. No entanto, o ganho genético materno foi negativo, sendo este dos anos de 2012 a 2016 de $-0,034 \mathrm{~kg}\left(\mathrm{R}^{2}=0,30\right)$. A variação fenotípica apresentou uma grande flutuação ao longo dos anos, em que no ano de 2013 o peso ao nascimento médio chegou aos 4,00 $\mathrm{kg}$, em 2015 atingiu os $3,88 \mathrm{~kg}$ e em 2016 o peso ao nascer médio chegou próximo à $3,97 \mathrm{~kg}$. Logo a variação dos anos de 2012 a 2016 foi positiva com valor na ordem de $0,032 \mathrm{~kg}\left(\mathrm{R}^{2}=0,04\right)$. Assim 
pode-se atribuir que neste rebanho, devido a menor influência ambiental na característica, o progresso genético é facilitado, uma vez determinando se a pressão seletiva será direcionada para maiores pesos ao nascimento, devido aos genes do feto, ou pela habilidade da ovelha como mãe, em fornecer um adequado ambiente uterino, proporcionando um maior crescimento fetal.

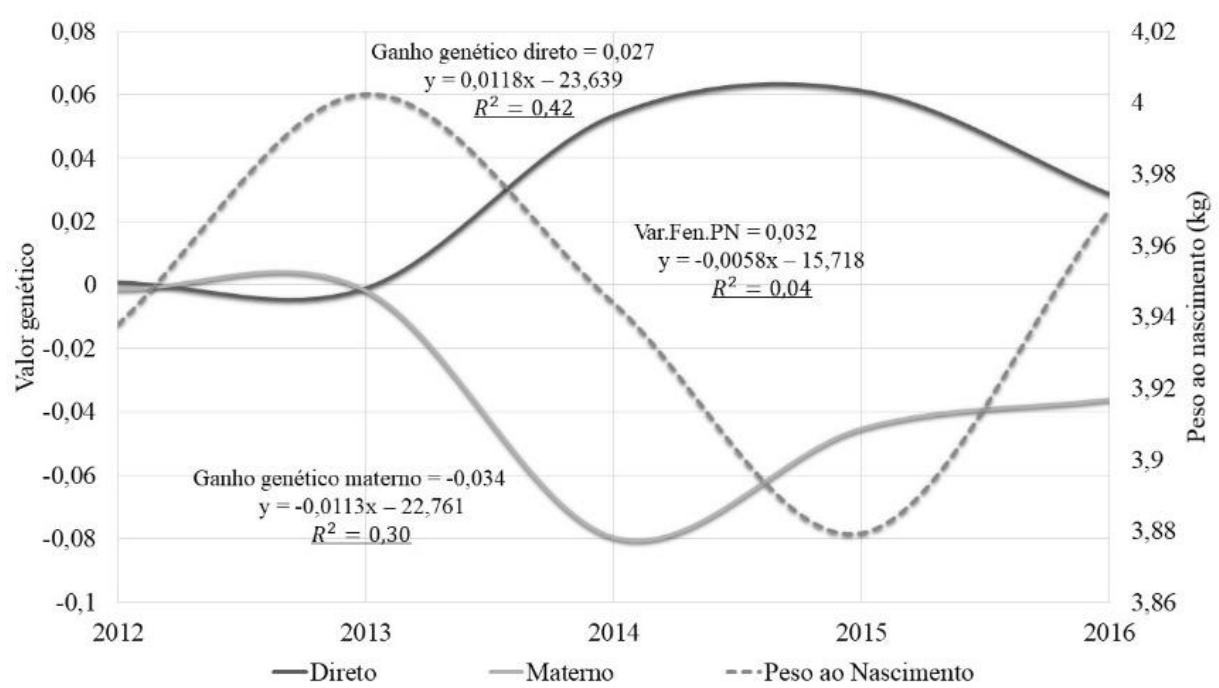

Figura 1. Tendências genética direta, materna e variação fenotípica para o peso ao nascimento, em ovinos cruza criados no Rio Grande do Sul.

Na raça Dorper, os ganhos genéticos direto e materno, entre 1982 a 1990, foram de 0,09 e 0,036 $\mathrm{kg}$, respectivamente (Neser et al., 2001). Para ovinos Columbia, de 1950 a 1998, o ganho genético direto foi de $0,8 \mathrm{~kg}$ (Hanford et al., 2002). Nesse mesmo período (1950-1998), na a raça Targhee, o ganho genético direto foi de $0,5 \mathrm{~kg}$. (Hanford et al., 2003). Em outro estudo com a raça Dorper, dos anos de 1984 a 1995, o ganho genético direto foi de $-0,150 \mathrm{~kg}$ (Kariuki et al., 2010). Mohammadi et al. (2013) avaliando ovinos Makooei, obtiveram ganhos genéticos direto e materno de 0,115 e 0,099, respectivamente, dos anos de 1996 a 2009. Para ovinos Baluchi, entre os anos de 1983 a 2010, Gholizadeh e Ghafouri-Kesbi (2015) não obtiveram progresso genético para o peso ao nascimento, obtendo ganho genético igual a $0,000 \mathrm{~kg}$. No entanto, nesse estudo com a raça Baluchi, os autores não discutem esse progresso genético nulo. Logo esse resultado deve ser devido a não utilização do peso ao nascer como critério de seleção.

Na raça Santa Inês, Aguirre et al. (2016), encontraram, de 2003 a 2014, um ganho genético de $-0,0132 \mathrm{~kg}$. Boujenane e Diallo et al. (2017) avaliando ovinos da raça Sardi, ao longo de 21 anos (1982-2004) calcularam ganhos genéticos direto de $-0,024 \mathrm{~kg}$ e materno de $0,023 \mathrm{~kg}$.

Assim, no presente estudo, pode-se inferir que o impacto do ganho direto, devido uma correlação negativa de - 0,69 entre os efeitos direto e materno, tenha ocasionado em uma supressão no progresso dos valores maternos, principalmente após o ano de 2013. Provavelmente estes obtidos podem ser atribuídos aos acasalamentos, direcionados ou ao acaso, de animais com maiores valores genéticos direto para a característica.

\section{Conclusão}

Para a estimativas dos parâmetros genéticos e cálculos de tendências genéticas mais acurados, deve-se lançar mão do modelo que considere os efeitos direto, materno e a covariância entre ambos. Neste sentido, com o modelo 9, verifica-se baixos coeficientes de herdabilidade direta e altos para o efeito materno, como uma alta correlação negativa entre os efeitos direto e materno. Assim, os ganhos genéticos diretos são positivos e os ganhos maternos negativos, sustentados por coeficientes de determinação de 0,42 e 0,30 , respectivamente.

\section{Conflito de Interesse}

Os autores declaram não existir conflito de interesse.

\section{Comitê de Ética}

Em relação aos aspectos éticos na experimentação animal, o presente trabalho não apresenta implicações que competem à apreciação da comissão de ética da instituição (Comissão de Ética em Experimentação Animal - CEEA), uma vez que os dados trabalhados foram cedidos pelos 
proprietários, que praticam tais registros, de maneira rotineira.

\section{Referências}

Abbasi, M.A.; Abdollahi-Arpanahi, R.; Maghsoudi, A.; Vaez Torshizi, R.; NejatiJavaremi, A. Evaluation of models for estimation of genetic parameters and maternal effects for early growth traits of Iranian Baluchi sheep. Small Ruminant Research, 104(3): 6269, 2012.

Aguirre, E.L.; Mattos, E.C.; Eler, J.P.; Barreto Neto, A.D.; Ferraz, J.B. Estimation of genetic parameters and genetic changes for growth characteristics of Santa Ines sheep. Genetics and Molecular Research, 15(3), 2016.

Akaike, K. On entropy maximization principle. In: Krishnaiah, P.R. Applications of statistics. Amsterdam: North Holland Publishing Co., 1977.

Amarilho-Silveira, F.; Dionello, N.J.L.; Mendonça, G.; Motta, J.F.; Weber, A.G. Parâmetros genéticos do peso total de cordeiros nascidos por ovelha em ovinos Texel. Revista Científica Rural, 19(2): 226-238, 2017.

Amarilho-Silveira, F.; Dionello, N.J.L.; Mendonça, G.; Motta, J.F.; Fernandes, T.A.; Silva, N.S. Genetic components of birth weight of Texel sheep reared in extensive system. Acta Scentarium, 40: e36481, 2018.

ARCO, Associação Brasileira de Criadores de Ovinos. Padrões raciais - ideal. Disponível em:

$<$ http://www.arcoovinos.com.br/sitenew/racas links/ideal.htm>. Acesso em: 18 de jul. 2016.

Bahreini Behzadi, M.R.; Shahroudi, F.E.; Van Vleck, L.D. Estimates of genetic parameters for growth traits in Kermani sheep. Journal of Animal Breeding and Genetics, 124(5): 296301, 2007.

Banchero, G.; Ganzábal, A.; Montossi, F.; Barbieri, I.; Quintans, G. Aportes de la Investigación para el Aumento de la Producción de Corderos. Veterinaria (Montevideo), 48(1): 13-18, 2012.

Boujenane, I.; Chikhi, A.; Ibnelbachyr, M.; Mouh, F.Z. Estimation of genetic parameters and maternal effects for body weight at different ages in D'man sheep. Small Ruminant Research, 130(1): 27-35, 2015.

Boujenane, I.; Diallo, I.T. Estimates of genetic parameters and genetic trends for pre-weaning growth traits in Sardi sheep. Small Ruminant Research, 146(1): 61-68, 2017.

Ceyhan, A.; Moore, K.; Mrode, R. The estimation of (co)variance components growth, reproduction, carcass, FECS and FECN traits in Lleyn sheep. Small Ruminant Research, 131(1): 29-34, 2015.

Ciappesoni, G.; Gimeno, D.; Coronel, F. Progreso genético logrado en las evaluaciones ovinas del Uruguay. Archivos Latinoamericanos de Producción Animal, 22(3, 4): 73-80, 2014.

Di, J.; Zhang, Y.; Tian, K.; Lazate; Liu, J.; Xu, X.; Zhang, Y.; Zhang, T. Estimation of (co)variance components and genetic parameters for growth and wool traits of Chinese superfine merino sheep with the use of a multi-trait animal model. Livestock Science, 138(1, 3): 278-288, 2011.

Eskandarinasab, M.; Ghafouri-Kesbi, F.; Abbasi, M.A. Different models for evaluation of growth traits and Kleiber ratio in an experimental flock of Iranian fat-tailed Afshari sheep. Journal of Animal Breeding and Genetics, 127(1): 2633, 2010.

Everett-Hincks, J.M.; Mathias-Davis, H.C.; Greer, G.J.; Auvray, B.A.; Dodds, K.G. Genetic parameters for lamb birth weight, survival and death risk traits. Journal of Animal Science, 92(7): 2885-2895, 2014.

Ganzábal, A.; Ciappesoni, G.; Banchero, G.; Vazquez, A.; Ravagnolo, O.; Montossi, F. Biotipos Maternales y Terminales para Enfrentar los Nuevos Desafíos de la Producción Ovina Moderna. Revista INIA, Producción Animal, 29(1): 14-18, 2012.

Gholizadeh, M.; Ghafouri-Kesbi, F. Estimation of genetic parameters for growth-related traits and evaluating the results of a 27 -year selection program in Baluchi sheep. Small Ruminant Research, 130(1): 8-14, 2015.

Gowane, G.R.; Chopra, A.; Prince, L.L.; Paswan, C.; Arora, A.L. Estimates of (co)variance components and genetic parameters for body weights and first greasy fleece weight in Bharat Merino sheep. Animal, 4(3): 425-431, 2010a.

Gowane, G.R.; Chopra, A.; Prakash, V.; Arora, A.L. Estimates of (co)variance components and genetic parameters for body weights and first greasy fleece weight in Malpura sheep. Livestock Science, 131(1): 94-101, 2010b.

Hanford, K.J.; Van Vleck, L.D.; Snowder, G.D. Estimates of genetic parameters and genetic 
change for reproduction, weight, and wool characteristics of Columbia sheep. Journal of Animal Science, 80(12): 3086-3098, 2002.

Hanford, K.J.; Van Vleck, L.D.; Snowder, G.D. Estimates of genetic parameters and genetic change for reproduction, weight, and wool characteristics of Targhee sheep. Journal of Animal Science, 81(3): 630-640, 2003.

Jafaroghli, M.; Rashidi, A.; Mokhtari, M.S.; Shadparvar, A.A. (Co)Variance components and genetic parameter estimates for growth traits in Moghani sheep. Small Ruminant Research, 91(2, 3): 170-177, 2010.

Kariuki, C.M.; Ilatsia, E.D.; Kosgey, I.S.; Kahi, A.K. Direct and maternal (co)variance components, genetic parameters and annual trends for growth traits of Dorper sheep in semiarid Kenya. Tropical Animal Health and Production, 42(3): 473-481, 2010.

Lavvaf, A.; Noshary, A. Estimation of genetic parameters and environmental factors on early growth traits for Lori breed sheep using single trait animal model. Pakistan Journal of Biological Sciences, 11(1): 74-79, 2008.

Lôbo, A.M.; Lôbo, R.N.; Paiva, S.R.; de Oliveira, S.M.; Facó, O. Genetic parameters for growth, reproductive and maternal traits in a multibreed meat sheep population. Genetics and Molecular Biology, 32(4): 761-770, 2009.

Lôbo, R.N.; Lôbo, A.M.; Facó, O. Melhoramento Genético de Ovinos. In: Selaive, A.B.; Osório, J.C.S. Produção de ovinos no Brasil, $1^{a}$ ed. São Paulo: Roca, 2014. p. 264-307.

Mandal, A.; Karunakaran, M.; Sharma, D.K.; Baneh, H.; Rout, P.K. Variance components and genetic parameters of growth traits and Kleiber ratio in Muzaffarnagari sheep. Small Ruminant Research, 132(1): 79-85, 2015.

Mcmanus, C.; Hermuche, P.; Paiva, S.; Silva, F.; Moraes, J.; Melo, C.; Mendes, C. Associação Brasileira de Criadores de Ovinos. Distribuição geográfica de raças de ovinos no Brasil e sua relação com fatores ambientais e climáticos, como a classificação de risco para a conservação. Artigos técnicos. Disponível em:http://www.arcoovinos.com.br/sitenew/ferr amenta/imagens/artigos/4.pdf>. Acesso em: 18 jul. 2016.

Meyer, K. WOMBAT, A program for Mixed Model Analyses by Restricted Maxi-mum Likelihood. User Notes. Animal Genetics and Breeding Unit, University of New England Armidale, Australia, 2016. 126p.
Mirhoseini, S.Z.; Zare, J.; Hossein-Zadeh, N.G.; Khanzadeh, H.; Seidavi, A.; Laudadio, V.; Dario, C.; Tufarelli, V.; Selvaggi, M. Estimation of genetic parameters for body weight traits and pelt quality score in Iranian Karakul sheep. Small Ruminant Research, 132(1): 67-71, 2015.

Mohammadi, H.; Edriss, M.A. Genetic parameters of early growth traits in Mehraban breed of sheep. Pakistan Journal of Biological Sciences, 10(3): 373-377, 2007.

Mohammadi, H.; Shahrebabak, M.M.; Vatankhah, M.; Shahrebabak, H.M. Direct and maternal (co)variance components, genetic parameters, and annual trends for growth traits of Makooei sheep in Iran. Tropical Animal Health and Production, 45(1): 185-191, 2013.

Mohammadi, K.; Abdollahi-Arpanahi, R.; Amraei, F.; Mohamadi, E.M.; Rashidi, A. Genetic parameter estimates for growth and reproductive traits in Lori sheep. Small Ruminant Research, 131(1): 35-42, 2015.

Neser, F.W.; Erasmus, G.J.; van Wyk, J.B. Genetic parameter estimates for pre-weaning weight traits in Dorper sheep. Small Ruminant Research, 40(3): 197-202, 2001.

Prakash, V.; Prince, L.L.L.; Gowane, G.R.; Arora, A.L. The estimation of (co)variance components and genetic parameters for growth traits and Kleiber ratios in Malpura sheep of India. Small Ruminant Research, 108(1, 3): 54-58, 2012.

R Core Team. R: A language and environment for statistical computing. $\mathbf{R}$ Foundation for Statistical Computing, Vienna, Austria. Disponível em: <https://www.R-project.org/.> Acessado em: 09 de jul. 2018.

Safari, E.; Fogarty, N.; Gilmour, A. A review of genetic parameter estimates for wool, growth, meat and reproduction traits in sheep. Livestock Production Science, 92(1): 271289, 2005.

Sawalha, R.; Conington, J.; Brotherstone, S.; Villanueva, B. Analysis of lamb survival of Scottish Blackface sheep. Animal, 1(1):151157, 2007.

Shiotsuki, L.; Oliveira, D.P.; Lôbo, R.N.B.; Facó, O. Genetic parameters for growth and reproductive traits of Morada Nova sheep kept by smallholder in semi-arid Brazil. Small Ruminant Research, 120(1): 204-208, 2014.

Shokrollahi, B.; Baneh, H. (Co)variance components and genetic parameters for growth 
traits in Arabi sheep using different animal models. Genetics and Molecular Research, 11(1): 305-314, 2012.

Ulutas, Z.; Sirin, E.; Aksoy, Y.; Sahin, A.; Kuran, M. Estimates of (co)variance components for direct and maternal effects on birth weight of Karayaka lambs. Tropical Animal Health and Production, 45(4): 953-956, 2013.
Zamani, P.; Mohammadi, H. Comparison of different models for estimation of genetic parameters of early growth traits in the Mehraban sheep. Journal of Animal Breeding and Genetics, 125(1): 29-34, 2008.

Zishiri, O.T.; Cloete, S.W.P.; Olivier, J.J.; Dzama, $\mathrm{K}$. Genetic parameters for live weight traits in South African terminal sire sheep breeds. Small Ruminant Research, 116(2,3): 118-125, 2014 